\title{
Optimal Control of a Rigid Body using Geometrically Exact Computations on $\mathrm{SE}(3)$
}

\author{
Taeyoung Lee*†, N. Harris McClamroch ${ }^{\dagger}$ \\ Department of Aerospace Engineering \\ University of Michigan, Ann Arbor, MI 48109 \\ $\{$ tylee, nhm\}@umich.edu
}

\author{
Melvin Leok* \\ Department of Mathematics \\ Purdue University, West Lafayette, IN 47907 \\ mleok@math.purdue.edu
}

\begin{abstract}
Optimal control problems are formulated and efficient computational procedures are proposed for combined orbital and rotational maneuvers of a rigid body in three dimensions. The rigid body is assumed to act under the influence of forces and moments that arise from a potential and from control forces and moments. The key features of this paper are its use of computational procedures that are guaranteed to preserve the geometry of the optimal solutions. The theoretical basis for the computational procedures is summarized, and examples of optimal spacecraft maneuvers are presented.
\end{abstract}

\section{INTRODUCTION}

Discrete optimal control problems for translational and rotational dynamics of a rigid body under a potential are studied. Optimal control of a rigid body arises in numerous engineering and scientific fields. These problems provide both a theoretical challenge and a numerical challenge in the sense that the configuration space has a Lie group structure denoted by $\mathrm{SE}(3)$ that defines a fundamental constraint.

Optimal control problems on a Lie group have been studied in [1], [2]. These studies are based on the driftless kinematics of a Lie group. The dynamics are ignored, and it is assumed that elements in the corresponding Lie algebra are controlled directly.

General-purpose numerical integration methods, including the popular Runge-Kutta schemes, typically preserve neither the group structure of the configuration space nor geometric invariants of the dynamics. Geometric structurepreserving integrators, referred to as Lie group variational integrators [3], preserve the group structure without the use of local charts, reprojection, or constraints, and they have the desirable property that they are symplectic and momentum preserving, and they exhibit good energy behavior for an exponentially long time period.

This paper presents geometrically exact and numerically efficient computational approaches to solve optimal control problems of a rigid body on a Lie group, $\mathrm{SE}(3)$. The dynamics and the kinematics are discretized by a Lie group variational integrator, and discrete optimality conditions are constructed. Efficient numerical algorithms to solve the necessary condition are developed. This method provide a substantial advantage over current methods for optimal

\footnotetext{
* This research has been supported in part by NSF under grant DMS0504747, and by a grant from the Rackham Graduate School, University of Michigan.

${ }^{\dagger}$ This research has been supported in part by NSF under grant ECS0244977.
}

control on a Lie group in the sense that the dynamics of a rigid body as well as the kinematics equation are explicitly utilized, and the proposed computational approaches respect the group structure.

This paper is organized as follows. In Section II, a Lie group variational integrator is developed. Optimal control problems using impulsive controls are studied in Section III, and optimal control problems with smooth controls are studied in Section IV. Numerical results for a rigid dumbbell spacecraft are given in Section V.

\section{LIE GROUP VARIATIONAL INTEGRATOR ON SE(3)}

The configuration space for the translational and rotational motion of a rigid body is the special Euclidean group, $\mathrm{SE}(3)=\mathbb{R}^{3} \mathrm{~S} \mathrm{SO}(3)$. We identify the cotangent bundle $\mathrm{T}^{*} \mathrm{SE}(3)$ with $\mathrm{SE}(3) \times \mathfrak{s e}(3)^{*}$ by left translation, and we identify $\mathfrak{s e}(3)^{*}$ with $\mathbb{R}^{6}$ by an isomorphism between $\mathbb{R}^{6}$ and $\mathfrak{s e}(3)$, and the standard inner product on $\mathbb{R}^{6}$. We denote the attitude, position, angular momentum, and linear momentum of the rigid body by $(R, x, \Pi, \gamma) \in \mathrm{T}^{*} \mathrm{SE}(3)$.

The continuous equations of motion are given by

$$
\begin{gathered}
\dot{x}=\frac{\gamma}{m}, \\
\dot{\gamma}=f+u^{f}, \\
\dot{R}=R S(\Omega), \\
\dot{\Pi}+\Omega \times \Pi=M+u^{m},
\end{gathered}
$$

where $\Omega \in \mathbb{R}^{3}$ is the angular velocity, and $u^{f}, u^{m} \in \mathbb{R}^{3}$ are the control force in the inertial frame and the control moment in the body fixed frame, respectively. The constant mass of the rigid body is $m \in \mathbb{R}$, and $J \in \mathbb{R}^{3 \times 3}$ denotes the moment of inertia, i.e. $\Pi=J \Omega$. The map $S(\cdot): \mathbb{R}^{3} \mapsto \mathfrak{s o}(3)$ is an isomorphism between $\mathfrak{s o}(3)$ and $\mathbb{R}^{3}$ defined by the condition $S(x) y=x \times y$ for all $x, y \in \mathbb{R}^{3}$.

We assume that the potential is dependent on the position and the attitude; $U(\cdot): \operatorname{SE}(3) \mapsto \mathbb{R}$. The corresponding force and the moment due to the potential are given by

$$
\begin{aligned}
f & =-\frac{\partial U}{\partial x}, \\
M & =r_{1} \times u_{r_{1}}+r_{2} \times u_{r_{2}}+r_{3} \times u_{r 3},
\end{aligned}
$$

where $r_{i}, u_{r_{i}} \in \mathbb{R}^{3}$ are the $i$ th row vector of $R$ and $\frac{\partial U}{\partial R}$, respectively.

Since the dynamics of a rigid body has the structure of a Lagrangian or Hamiltonian system, they are characterized 
by symplectic, momentum and energy preserving properties. These geometric features determine the qualitative behavior of the rigid body dynamics, and they can serve as a basis for theoretical study of rigid body dynamics.

In contrast, the most common numerical integration methods, including the widely used Runge-Kutta schemes, neither preserve the Lie group structure nor these geometric properties. In addition, standard Runge-Kutta methods fail to capture the energy dissipation of a controlled system accurately [4]. Additionally, if we integrate (3) by a typical Runge-Kutta scheme, the quantity $R^{T} R$ inevitably drifts from the identity matrix as the simulation time increases. It is often proposed to parameterize (3) by Euler angles or unit quaternions. However, Euler angles are not global expressions of the attitude since they have associated singularities. Unit quaternions do not exhibit singularities, but are constrained to lie on the unit three-sphere $\mathbb{S}^{3}$, and general numerical integration methods do not preserve the unit length constraint. Therefore, quaternions have the same numerical drift problem. Renormalizing the quaternion vector at each step tends to break other conservation properties. Furthermore, unit quaternions, which are diffeomorphic to $\mathrm{SU}(2)$, double cover $\mathrm{SO}(3)$. So there are inevitable ambiguities in expressing the attitude.

In [3], Lie group variational integrators are introduced by explicitly adapting Lie group methods [5] to the discrete variational principle [4]. They have the desirable property that they are symplectic and momentum preserving, and they exhibit good energy behavior for an exponentially long time period. They also preserve the Euclidian Lie group structure without the use of local charts, reprojection, or constraints. These geometrically exact numerical integration methods yield highly efficient and accurate computational algorithms for rigid body dynamics. They avoid singularities and ambiguities.

Using the results presented in [6], a Lie group variational integrator on $\mathrm{SE}(3)$ for equations (1)-(4) is given by

$$
\begin{gathered}
x_{k+1}=x_{k}+\frac{h}{m} \gamma_{k}+\frac{h^{2}}{2 m}\left(f_{k}+u_{k}^{f}\right) \\
\gamma_{k+1}=\gamma_{k}+\frac{h}{2}\left(f_{k}+u_{k}^{f}\right)+\frac{h}{2}\left(f_{k+1}+u_{k+1}^{f}\right) \\
h S\left(\Pi_{k}+\frac{h}{2}\left(M_{k}+u_{k}^{m}\right)\right)=F_{k} J_{d}-J_{d} F_{k}^{T} \\
R_{k+1}=R_{k} F_{k}, \\
\Pi_{k+1}=F_{k}^{T} \Pi_{k}+\frac{h}{2} F_{k}^{T}\left(M_{k}+u_{k}^{m}\right)+\frac{h}{2}\left(M_{k+1}+u_{k+1}^{m}\right)
\end{gathered}
$$

where the subscript $k$ denotes the $k$ th discrete variables for a fixed integration step size $h \in \mathbb{R}$. $J_{d} \in \mathbb{R}^{3 \times 3}$ is a nonstandard moment of inertia matrix defined by $J_{d}=$ $\frac{1}{2} \operatorname{tr}[J] I_{3 \times 3}-J . F_{k} \in \mathrm{SO}(3)$ is the relative attitude between adjacent integration steps.

For given $\left(R_{k}, x_{k}, \Pi_{k}, \gamma_{k}\right)$ and control inputs, (9) is solved to find $F_{k}$. Then $\left(R_{k+1}, x_{k+1}\right)$ are obtained by (10),(7). Using (5),(6), $\left(f_{k+1}, M_{k+1}\right)$ are computed, and they are used to find $\left(\Pi_{k+1}, \gamma_{k+1}\right)$ by $(11),(8)$. This yields a map $\left(R_{k}, x_{k}, \Pi_{k}, \gamma_{k}\right) \mapsto\left(R_{k+1}, x_{k+1}, \Pi_{k+1}, \gamma_{k+1}\right)$, and this process is repeated. The only implicit part is (9). The actual computation of $F_{k}$ is done in the Lie algebra $\mathfrak{s o}(3)$ of dimension 3 , and the rotation matrices are updated by multiplication. This approach is completely different from integration of the kinematics equation (3); there is no excessive computational burden. It can be shown that this integrator has second order accuracy. The properties of these discrete equations of motion are discussed in more detail in [3], [6].

\section{Optimal Impulsive CONTROL OF A Rigid Body}

We formulate an optimal impulsive control problem for a rigid body on $\mathrm{SE}(3)$, and we develop sensitivity derivatives. They are used in our computational method for solve optimal impulsive control problems.

\section{A. Problem formulation}

An optimal impulsive control problem is formulated as a maneuver of a rigid body from a given initial configuration $\left(R_{0}, x_{0}, \Pi_{0}, \gamma_{0}\right)$ to a desired configuration described by

$\left\{\left(R_{N}, x_{N}, \Pi_{N}, \gamma_{N}\right) \in \mathrm{T}^{*} \mathrm{SE}(3) \mid \mathcal{C}\left(R_{N}, x_{N}, \Pi_{N}, \gamma_{N}\right)=0\right\}$, where $\mathcal{C}(\cdot): \mathrm{T}^{*} \mathrm{SE}(3) \mapsto \mathbb{R}^{c}$ during the given maneuver time $N$. Two impulsive control inputs are applied at the initial time and the terminal time. We assume that the control inputs are purely impulsive, which means that each impulse changes the momentum of the rigid body instantaneously, but it does not have any effect on the position and the attitude of the rigid body at that instant. The motion of the rigid body between the initial time and the terminal time is uncontrolled. i.e. $u_{k}^{f}=u_{k}^{m}=0$. The performance index is the sum of the magnitudes of the initial impulse and the terminal impulse. It is equivalent to minimizing the sums of the initial momentum change and the terminal momentum change.

We transform this optimal impulsive control problem into a parameter optimization problem. Let $\left(\Pi_{0}^{+}, \gamma_{0}^{+}\right)$be the initial momentum after the initial impulsive control. Then, the terminal states are determined by the discrete equations of motion, and the momentum after the terminal impulsive control, $\left(\Pi_{N}^{+}, \gamma_{N}^{+}\right)$, can be computed by the terminal constraint. Therefore, the performance index and the constraint are completely determined by $\left(\Pi_{0}^{+}, \gamma_{0}^{+}\right)$. Thus, the optimal impulsive control on $\mathrm{SE}(3)$ is formulated as

$$
\begin{gathered}
\text { given : }\left(R_{0}, x_{0}, \Pi_{0}, \gamma_{0}\right), N \\
\min _{\Pi_{0}^{+}, \gamma_{0}^{+}} \mathcal{J}=\left\|\Pi_{0}^{+}-\Pi_{0}\right\|+\left\|\gamma_{0}^{+}-\gamma_{0}\right\| \\
+\left\|\Pi_{N}^{+}-\Pi_{N}\right\|+\left\|\gamma_{N}^{+}-\gamma_{N}\right\|, \\
\text { such that } \mathcal{C}\left(R_{N}, x_{N}, \Pi_{N}^{+}, \gamma_{N}^{+}\right)=0,
\end{gathered}
$$

subject to discrete equations of motion (7)-(11).

If the desired values for all of the terminal states are specified by the constraints, then there is no freedom for optimization. This problem degenerates to a two point boundary value problem on $\mathrm{SE}(3)$, which can be considered as an extension of the Lambert problem for the restricted two body problem. A similar optimal control problem for attitude dynamics of a rigid body on $\mathrm{SO}(3)$ is studied in [7]. 


\section{B. Sensitivity derivatives}

Variational model: The variation of $g_{k}=\left(R_{k}, x_{k}\right) \in$ $\mathrm{SE}(3)$ can be expressed in terms of a Lie algebra element $\eta_{k} \in \mathfrak{s e}(3)$ and the exponential map as $g_{k}^{\epsilon}=g_{k} \exp \epsilon \eta_{k}$. The corresponding infinitesimal variation is given by

$$
\delta g_{k}=\left.\frac{d}{d \epsilon}\right|_{\epsilon=0} g_{k} \exp \epsilon \eta_{k}=\mathrm{T}_{e} L_{g_{k}} \cdot \eta_{k} .
$$

Using homogeneous coordinates [8], the above equation is written in a matrix equation as

$$
\begin{aligned}
{\left[\begin{array}{cc}
\delta R_{k} & \delta x_{k} \\
0 & 0
\end{array}\right] } & =\left[\begin{array}{cc}
R_{k} & x_{k} \\
0 & 1
\end{array}\right]\left[\begin{array}{cc}
S\left(\zeta_{k}\right) & \chi_{k} \\
0 & 0
\end{array}\right], \\
& =\left[\begin{array}{cc}
R_{k} S\left(\zeta_{k}\right) & R_{k} \chi_{k} \\
0 & 0
\end{array}\right],
\end{aligned}
$$

where $\zeta_{k}, \chi_{k} \in \mathbb{R}^{3}$ so that $\left(S\left(\zeta_{k}\right), \chi_{k}\right) \in \mathfrak{s e}(3)$. This gives an expression for the infinitesimal variation of a Lie group element in terms of its Lie algebra. Then, small perturbations from a given trajectory on $\mathrm{T}^{*} \mathrm{SE}(3)$ can be written as

$$
\begin{aligned}
x_{k}^{\epsilon} & =x_{k}+\epsilon \delta x_{k}, \\
\gamma_{k}^{\epsilon} & =\gamma_{k}+\epsilon \delta \gamma_{k}, \\
\Pi_{k}^{\epsilon} & =\Pi_{k}+\epsilon \delta \Pi_{k}, \\
R_{k}^{\epsilon} & =R_{k}+\epsilon R_{k} S\left(\zeta_{k}\right)+\mathcal{O}\left(\epsilon^{2}\right),
\end{aligned}
$$

where $\delta x_{k}, \delta \gamma_{k}, \delta \Pi_{k}, \zeta_{k}$ are considered in $\mathbb{R}^{3}$.

We derive expressions for the constrained variation of $F_{k}$ using (10) and (16). Since $F_{k}=R_{k}^{T} R_{k+1}$ by (10), the infinitesimal variation $\delta F_{k}$ is given by

$$
\delta F_{k}=\delta R_{k}^{T} R_{k+1}+R_{k}^{T} \delta R_{k+1}=-S\left(\zeta_{k}\right) F_{k}+F_{k} S\left(\zeta_{k+1}\right) .
$$

We can also express $\delta F_{k}=F_{k} S\left(\xi_{k}\right)$ for $\xi_{k} \in \mathbb{R}^{3}$, using (12). Using the property $S\left(R^{T} x\right)=R^{T} S(x) R$ for all $R \in \mathrm{SO}(3)$ and $x \in \mathbb{R}^{3}$, we obtain the constrained variation of $F_{k}$

$$
\xi_{k}=-F_{k}^{T} \zeta_{k}+\zeta_{k+1} .
$$

Linearized equations of motion: Substituting the variation model (13)-(16) and the constrained variation (17) into the equations of motion (7)-(11), and ignoring higher order terms, the linearized equation of motion can be written as

$$
z_{k+1}=A_{k} z_{k}
$$

where $z_{k}=\left[\delta x_{k} ; \delta \gamma_{k} ; \zeta_{k} ; \delta \Pi_{k}\right] \in \mathbb{R}^{12}$, and $A_{k} \in \mathbb{R}^{12 \times 12}$ can be suitably defined. The solution of (18) is obtained as

$$
z_{N}=\Phi z_{0}
$$

where $\Phi \in \mathbb{R}^{12 \times 12}$ represents the sensitivity derivatives of the terminal state with respect to the initial state on $\mathrm{SE}(3)$.

\section{Computational approach}

We solve the optimal impulsive control problem by the Sequential Quadratic Programming (SQP) method using analytical expressions for the gradients of the performance index and the constraints. The exact computation of the gradients are crucial for efficient numerical optimization. For the given problem, $\delta x_{0}=\zeta_{0}=0$ since the initial position and the initial attitude are fixed. Thus, (19) is written as

$$
\left[\begin{array}{c}
\delta x_{N} \\
\delta \gamma_{N} \\
\zeta_{N} \\
\delta \Pi_{N}
\end{array}\right]=\left[\begin{array}{ll}
\Phi^{12} & \Phi^{14} \\
\Phi^{22} & \Phi^{24} \\
\Phi^{32} & \Phi^{34} \\
\Phi^{42} & \Phi^{44}
\end{array}\right]\left[\begin{array}{l}
\delta \gamma_{0}^{+} \\
\delta \Pi_{0}^{+}
\end{array}\right],
$$

where $\Phi^{i j} \in \mathbb{R}^{3 \times 3}, i, j \in(1,2,3,4)$ are submatrices of $\Phi$. The above equation represents the sensitivities of the terminal state with respect to the initial momentum $\left(\Pi_{0}^{+}, \gamma_{0}^{+}\right)$. Therefore, we can obtain expressions for gradients of the performance index and the constraints, and any Newton type numerical approach can be applied.

\section{Optimal Control of a Rigid Body}

We formulate an optimal control problem for a rigid body on $\mathrm{SE}(3)$ assuming that control forces and moments are applied during the maneuver. Necessary conditions for optimality are developed and computational approaches are presented to solve the corresponding two point boundary value problem.

\section{A. Problem formulation}

An optimal impulsive control problem is formulated as a maneuver of a rigid body from a given initial configuration $\left(R_{0}, x_{0}, \Pi_{0}, \gamma_{0}\right)$ to a desired configuration $\left(R_{N}^{d}, x_{N}^{d}, \Pi_{N}^{d}, \gamma_{N}^{d}\right)$ during the given maneuver time $N$. Control inputs are parameterized by their value at each time step. The performance index is the square of the weighted $l_{2}$ norm of the control inputs.

$$
\begin{gathered}
\text { given: }\left(x_{0}, \gamma_{0}, R_{0}, \Pi_{0}\right),\left(x_{N}^{d}, \gamma_{N}^{d}, R_{N}^{d}, \Pi_{N}^{d}\right), N, \\
\min _{u_{k+1}} \mathcal{J}=\sum_{k=0}^{N-1} \frac{h}{2}\left(u_{k+1}^{f}\right)^{T} W_{f} u_{k+1}^{f}+\frac{h}{2}\left(u_{k+1}^{m}\right)^{T} W_{m} u_{k+1}^{m},
\end{gathered}
$$

such that $\left(x_{N}, \gamma_{N}, R_{N}, \Pi_{N}\right)=\left(x_{N}^{d}, \gamma_{N}^{d}, R_{N}^{d}, \Pi_{N}^{d}\right)$,

subject to discrete equations of motion (7)-(11),

where $W_{f}, W_{m} \in \mathbb{R}^{3 \times 3}$ are symmetric positive definite matrices. Here we use a modified version of the discrete equations of motion with first order accuracy, because it yields a compact form for the necessary conditions, which are developed the following subsection. A similar optimal control problem for attitude dynamics on $\mathrm{SO}(3)$ is studied in [9].

\section{B. Necessary conditions for optimality}

Define an augmented performance index as

$$
\begin{aligned}
\mathcal{J}_{a}=\sum_{k=0}^{N-1} & \frac{h}{2}\left(u_{k+1}^{f}\right)^{T} W^{f} u_{k+1}^{f}+\frac{h}{2}\left(u_{k+1}^{m}\right)^{T} W^{m} u_{k+1}^{m} \\
& +\lambda_{k}^{1, T}\left\{-x_{k+1}+x_{k}+\frac{h}{m} \gamma_{k}\right\} \\
& +\lambda_{k}^{2, T}\left\{-\gamma_{k+1}+\gamma_{k}+h f_{k+1}+h u_{k+1}^{f}\right\} \\
& +\lambda_{k}^{3, T} S^{-1}\left(\operatorname{logm}\left(F_{k}-R_{k}^{T} R_{k+1}\right)\right) \\
& +\lambda_{k}^{4, T}\left\{-\Pi_{k+1}+F_{k}^{T} \Pi_{k}+h\left(M_{k+1}+u_{k+1}^{m}\right)\right\},
\end{aligned}
$$


where $\lambda_{k}^{i} \in \mathbb{R}^{3}$ are Lagrange multipliers. The constraint (9) is considered implicitly using a constrained variation. Using the variational model (13)-(16), the constrained variation (17), and the fact that the variations vanish at $k=0, N$, we obtain the infinitesimal variation of $\mathcal{J}_{a}$ as

$$
\begin{aligned}
\delta \mathcal{J}_{a} & =\sum_{k=1}^{N-1} h \delta u_{k}^{f, T}\left\{W_{f} u_{k}^{f}+\lambda_{k-1}^{2}\right\} \\
& +h \delta u_{k}^{m, T}\left\{W_{m} u_{k}^{m}+\lambda_{k-1}^{4}\right\}+z_{k}^{T}\left\{-\lambda_{k-1}+A_{k}^{T} \lambda_{k}\right\},
\end{aligned}
$$

where $\lambda_{k}=\left[\lambda_{k}^{1} ; \lambda_{k}^{2} ; \lambda_{k}^{3} ; \lambda_{k}^{4}\right] \in \mathbb{R}^{12}$, and $A_{k} \in \mathbb{R}^{12 \times 12}$ is presented in (18).

Since $\delta \mathcal{J}_{a}=0$ for all variations, we obtain necessary conditions for optimality as follows.

$$
\begin{gathered}
x_{k+1}=x_{k}+\frac{h}{m} \gamma_{k}, \\
\gamma_{k+1}=\gamma_{k}+h f_{k+1}+h u_{k+1}^{f}, \\
h S\left(\Pi_{k}\right)=F_{k} J_{d}-J_{d} F_{k}^{T}, \\
R_{k+1}=R_{k} F_{k}, \\
\Pi_{k+1}=F_{k}^{T} \Pi_{k}+h M_{k+1}+h u_{k+1}^{m}, \\
u_{k+1}^{f}=-W_{f}^{-1} \lambda_{k}^{2}, \\
u_{k+1}^{m}=-W_{m}^{-1} \lambda_{k}^{4}, \\
\lambda_{k}=A_{k+1}^{T} \lambda_{k+1} .
\end{gathered}
$$

In the above equations, the only implicit part is (23). For a given initial condition $\left(R_{0}, x_{0}, \Pi_{0}, \gamma_{0}\right)$ and $\lambda_{0}$, we can find $F_{0}$ by solving (23). Then, $R_{1}, x_{1}$ is obtained by (24),(21), and the control input $u_{1}^{f}, u_{1}^{m}$ is obtained by (26),(27). $\gamma_{1}, \Pi_{1}$ can be obtained by (22),(25). Now we compute $\left(R_{1}, x_{1}, \Pi_{1}, \gamma_{1}\right)$. We solve (23) to find $F_{1}$. Finally, $\lambda_{1}$ can be obtained by (28). This yields a map $\left\{\left(R_{0}, x_{0}, \Pi_{0}, \gamma_{0}\right), \lambda_{0}\right\} \mapsto$ $\left\{\left(R_{1}, x_{1}, \Pi_{1}, \gamma_{1}\right), \lambda_{1}\right\}$, and this process can be repeated.

\section{Computational Approach}

The necessary conditions for optimality are expressed in terms of a two point boundary problem on $\mathrm{T}^{*} \mathrm{SE}(3)$ and its dual. This problem is to find the optimal discrete flow, multiplier, and control inputs to satisfy the equations of motion (21)-(25), optimality conditions (26),(27), multiplier equations (28), and boundary conditions simultaneously.

We use a neighboring extremal method [10]. A nominal solution satisfying all of the necessary conditions except the boundary conditions is chosen. The unspecified initial multiplier is updated by successive linearization so as to satisfy the specified terminal boundary conditions in the limit. This is also referred to as a shooting method. The main advantage of the neighboring extremal method is that the number of iteration variables is small. In other approaches, the initial guess of control input history or multiplier variables are iterated, so the number of optimization parameters are proportional to the number of discrete time steps.

The difficulty is that the extremal solutions are sensitive to small changes in the unspecified initial multiplier values. The nonlinearities also make it hard to construct an accurate estimate of sensitivity, and it may result in numerical illconditioning. Therefore, it is important to compute the sensitivities accurately to apply the neighboring extremal method.

Here the optimality conditions (26) and (27) are substituted into the equations of motion and the multiplier equations. The sensitivities of the specified terminal boundary conditions with respect to the unspecified initial multiplier conditions is obtained by a linear analysis.

Similar to (18), the linearized equations of motion can be written as

$$
z_{k+1}=A_{k} z_{k}+\mathcal{A}^{12} \delta \lambda_{k}
$$

where $\mathcal{A}_{k}^{12}=-h \operatorname{diag}\left[0, W_{f}^{-1}, 0, W_{m}^{-1}\right] \in \mathbb{R}^{12 \times 12}$. We can linearize the multiplier equations (28) to obtain

$$
\delta \lambda_{k}=\mathcal{A}_{k+1}^{21} z_{k+1}+A_{k+1}^{T} \delta \lambda_{k+1},
$$

where $\mathcal{A}_{k+1}^{21} \in \mathbb{R}^{12 \times 12}$ can be defined properly. The solution of the linear equations (29) and (30) can be obtained as

$$
\left[\begin{array}{c}
z_{N} \\
\delta \lambda_{N}
\end{array}\right]=\left[\begin{array}{ll}
\Psi^{11} & \Psi^{12} \\
\Psi^{21} & \Psi^{22}
\end{array}\right]\left[\begin{array}{c}
z_{0} \\
\delta \lambda_{0}
\end{array}\right],
$$

where $\Psi^{i j} \in \mathbb{R}^{12 \times 12}$.

For the given two point boundary value problem $z_{0}=0$ since the initial condition is fixed, and $\lambda_{N}$ is free. Thus,

$$
z_{N}=\Psi_{12} \delta \lambda_{0}
$$

The matrix $\Psi_{12}$ represents the sensitivity of the specified terminal boundary conditions with respect to the unspecified initial multipliers. Using this sensitivity, an initial guess of the unspecified initial conditions is iterated to satisfy the specified terminal conditions in the limit.

Any type of Newton iteration can be applied. We use a line search with backtracking algorithm, referred to as NewtonArmijo iteration in [11]. The procedure is summarized as follows.

1: Guess an initial multiplier $\lambda_{0}$.

2: Find $x_{k}, \gamma_{k}, \Pi_{k}, R_{k}, \lambda_{k}$ using (21)-(28).

3: Compute the terminal B.C. error; Error $=\left\|z_{N}\right\|$.

4: Set $_{\text {Error }}{ }^{t}=$ Error, $i=1$.

5: while Error $>\epsilon_{S}$.

6: Find a line search direction; $D=\Psi_{12}^{-1}$.

7: $\quad$ Set $c=1$.

8: $\quad$ while Error $^{t}>(1-2 \alpha c)$ Error

9: $\quad$ Choose a trial multiplier $\lambda_{0}^{t}=\lambda_{0}+c D z_{N}$.

10: $\quad$ Find $x_{k}, \gamma_{k}, \Pi_{k}, R_{k}, \lambda_{k}$ using (21)-(28).

11: $\quad$ Compute the error; Error $^{t}=\left\|z_{N}^{t}\right\|$.

12: $\quad$ Set $c=c / 10, \quad i=i+1$.

13: end while

14: $\quad$ Set $\lambda_{0}=\lambda_{0}^{t}$, Error $=$ Error $^{t}$. (accept the trial)

15: end while

Here $i$ is the number of iterations, and $\epsilon_{S}, \alpha \in \mathbb{R}$ are a stopping criterion and a scaling factor, respectively. The outer loop finds a search direction by computing the sensitivity derivatives, and the inner loop performs a line search to find the largest step size $c \in \mathbb{R}$ along the search direction. The error in satisfaction of the terminal boundary condition is determined at each inner iteration. 


\section{NumERICAL EXAMPLES}

\section{A. Restricted Full Two Body Problem}

We study a maneuver of a rigid spacecraft under a central gravity field. We assume that the mass of the spacecraft is negligible compared to the mass of a central body, and we consider a fixed frame attached to the central body as an inertial frame. The resulting model is a Restricted Full Two Body Problem (RF2BP).

The spacecraft is modeled as a dumbbell, which consists of two equal spheres and a massless rod. The gravitational potential is given by

$$
U(x, R)=-\frac{G M m}{2} \sum_{q=1}^{2} \frac{1}{\left\|x+R \rho^{q}\right\|},
$$

where $G \in \mathbb{R}$ is the gravitational constant, $M, m \in \mathbb{R}$ are the mass of the central body, and the mass of the dumbbell, respectively. The vector $\rho^{q} \in \mathbb{R}^{3}$ is the position of the $q$ th sphere from the mass center of the dumbbell expressed in the body fixed frame $(q \in\{1,2\})$. The mass, length, and time dimensions are normalized by the mass of the dumbbell, the radius of a reference circular orbit, and its orbital period.

\section{B. Optimal Impulsive Control}

We study an impulsive orbital transfer problem with an attitude change. Initially, the spacecraft is on a reference circular orbit. We consider two cases. In the first case, the spacecraft moves to a desired circular orbit and the desired values for all of the terminal state are specified. There is no freedom for optimization, and the resulting problem is a two point boundary value problem on $\mathrm{SE}(3)$. This maneuver can be considered as a generalization of Hohmann transfer [12]. The desired maneuver involves doubling the orbital radius in addition to a large angle attitude change.

In the second case, the terminal constraints are relaxed such that the spacecraft is allowed to transfer to any point on the desired orbit. The desired terminal orbit is described by its orbital radius $r_{d} \in \mathbb{R}$, and a directional vector $e_{n} \in$ $\mathbb{S}^{2}$ normal to the orbital plane. Two constraints are imposed to locate the dumbbell in the desired orbital plane with the desired orbital radius, and one constraint is applied to align the dumbbell to the normal direction.

The gradients of the performance index and the constraints are obtained by using (20). We use Matlab fmincon function as an implementation of the SQP algorithm. Figures 1 and 2 show the spacecraft maneuver, and linear velocity and angular velocity responses, where red circles denote the velocities before the initial impulse and the velocities after the terminal impulse. Thus, differences between solid lines and red circles are proportional to the impulsive controls. (Simple animations which show these maneuvers of the spacecraft can be found at http://www.umich.edu/ $\sim$ tylee.) The error in satisfaction of the terminal boundary value of the first case is $4.77 \times 10^{-15}$. The performance index and the maximum violations of the constraints for the second case are 1.2305 and $3.88 \times 10^{-15}$, respectively.

\section{Optimal Control}

We study an optimal orbital transfer problem to increase the orbital inclination by $60 \mathrm{deg}$, and an orbital capture problem to the reference circular orbit.

Figures 3 and 4 show the optimized spacecraft maneuver, control inputs history. For each case, the performance indices are 13.03 and 20.90 , and the maximum violations of the constraint are $3.35 \times 10^{-13}$ and $3.26 \times 10^{-13}$, respectively.

Figures 3.(b) and 4.(b) show the violation of the terminal boundary condition according to the number of iterations in a logarithmic scale. Red circles denote outer iterations in Newton-Armijo iteration to compute the sensitivity derivatives. For all cases, the initial guesses of the unspecified initial multiplier are arbitrarily chosen. The error in satisfaction of the terminal boundary condition converges quickly to machine precision after the solution is close to the local minimum at around 20th iteration. These convergence results are consistent with the quadratic convergence rates expected of Newton methods with accurately computed gradients.

The neighboring extremal method, also referred to as the shooting method, is numerically efficient in the sense that the number of optimization parameters is minimized. But, this approach may be prone to numerical ill-conditioning [13]. A small change in the initial multiplier can cause highly nonlinear behavior of the terminal attitude and angular momentum. It is difficult to compute the gradient for Newton iterations accurately, and the numerical error may not converge.

However, the numerical examples presented in this paper show excellent numerical convergence properties. This is because the proposed computational algorithms on $\mathrm{SE}(3)$ are geometrically exact and numerically accurate.

The dynamics of a rigid body arises from Hamiltonian mechanics, which have neutral stability, and its adjoint system is also neutrally stable. The proposed Lie group variational integrator and the discrete multiplier equations, obtained from variations expressed in the Lie algebra, preserve the neutral stability property numerically. Therefore the sensitivity derivatives are computed accurately.

\section{CONCLUSIONS}

Optimal control problems for combined orbital and rotational maneuvers of a rigid body are formulated and efficient computational procedures are proposed. The dynamics are discretized by a Lie group variational integrator, and sensitivity derivatives are developed by a linear analysis. Discrete necessary conditions for optimality are constructed, and the corresponding two point boundary value problem is solved efficiently.

This approach is geometrically exact in the sense that the Lie group variational integrator preserves the group structure as well as the geometric invariant properties, and the sensitivity derivatives are expressed in terms of its Lie algebra. Since the configuration of a rigid body is defined globally using an element of $\mathrm{SE}(3)$, this approach completely avoids singularity or ambiguity arising from other representations such as Euler angles and quaternions. Numerical examples show the efficiency of the proposed computational approach. 


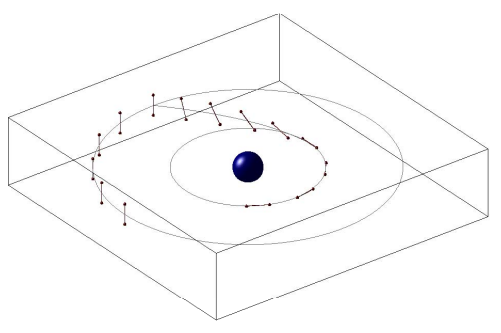

(a) Spacecraft maneuver

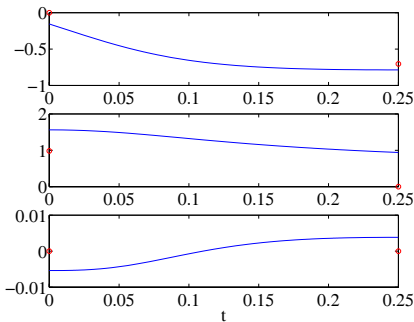

(b) Velocity $v$

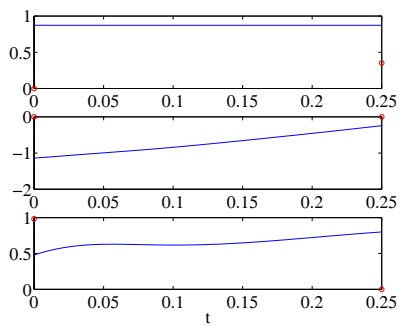

(c) Angular velocity $\Omega$
Fig. 1. TPBVP: Orbital radius change

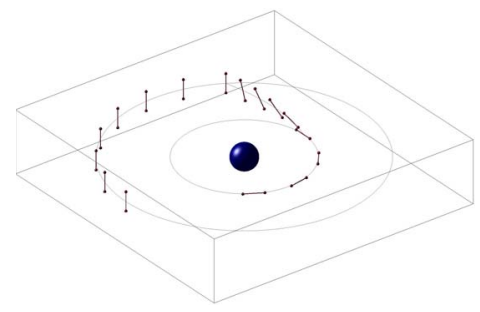

(a) Spacecraft maneuver

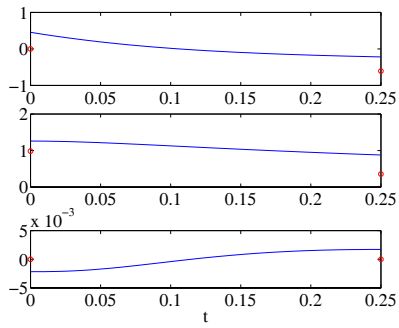

(b) Velocity $v$

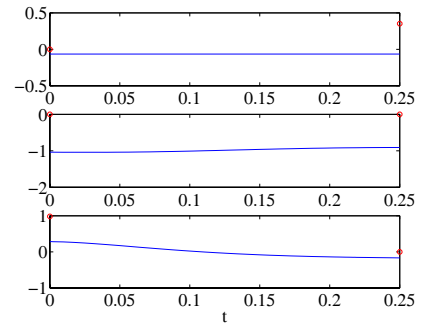

(c) Angular velocity $\Omega$
Fig. 2. Optimal impulsive control: Orbital radius change

\section{REFERENCES}

[1] K. Spindler, "Optimal control on Lie groups with applications to attitude control," Mathematics of Control, Signals, and Systems, vol. 11, pp. 197-219, 1998.

[2] S. Sastry, "Optimal control on Lie groups," in Proceedings of the Third International Congress on Industrial and Applied Mathematics (ICIAM), 1995.

[3] T. Lee, M. Leok, and N. H. McClamroch, "A Lie group variational integrator for the attitude dynamics of a rigid body with applications to the 3D pendulum," in Proceedings of the IEEE Conference on Control Applications, 2005, pp. 962-967.

[4] J. E. Marsden and M. West, "Discrete mechanics and variational integrators," Acta Numerica, vol. 10, pp. 357-514, 2001

[5] A. Iserles, H. Z. Munthe-Kaas, S. P. Nørsett, and A. Zanna, "Lie-group methods," Acta Numerica, vol. 9, pp. 215-365, 2000.

[6] T. Lee, M. Leok, and N. H. McClamroch, "Lie group variational integrators for the full body problem," Computer Methods in Applied

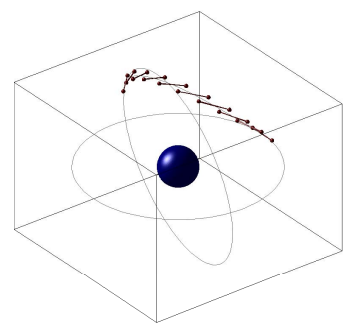

(a) Spacecraft maneuver

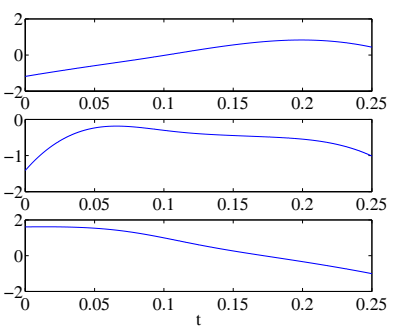

(c) Control force $u^{f}$

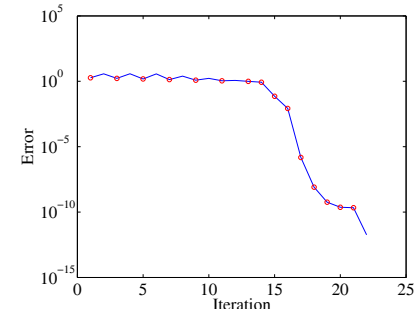

(b) Convergence rate

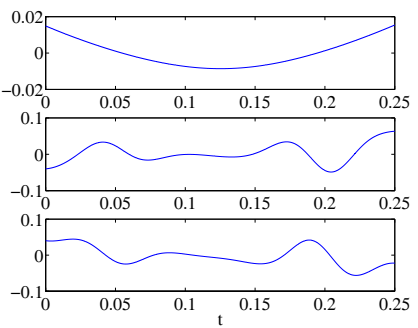

(d) Control moment $u^{m}$
Fig. 3. Optimal control: Orbital inclination change

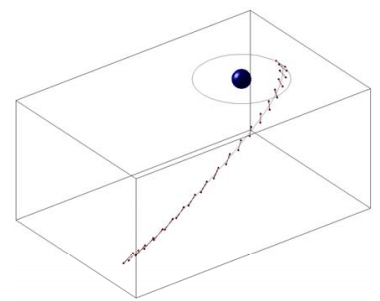

(a) Spacecraft maneuver

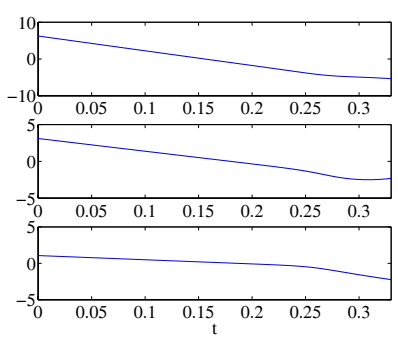

(c) Control force $u^{f}$

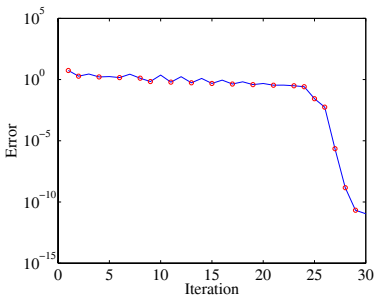

(b) Convergence rate

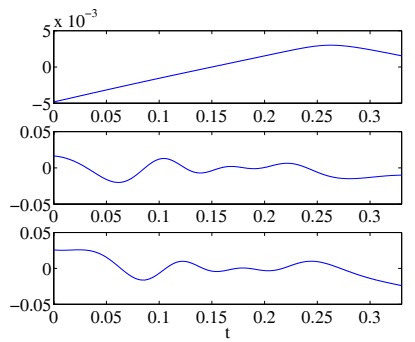

(d) Control moment $u^{m}$
Fig. 4. Optimal control: Orbital capture

Mechanics and Engineering, 2005, submitted. [Online]. Available: http://arxiv.org/math.NA/0508365

[7] _ "Attitude maneuvers of a rigid spacecraft in a circular orbit," in Proceedings of the American Control Conference, 2006, pp. 1742-1747. [Online]. Available: http://arxiv.org/math.NA/0509299

[8] R. M. Murray, Z. Li, and S. S. Sastry, A Mathematical Introduction to Robotic Manipulation. CRC Press, 1993.

[9] T. Lee, M. Leok, and N. H. McClamroch, "Optimal attitude control of a rigid body using geometrically exact computations on $\mathrm{SO}(3)$," Journal of Optimization Theory and Applications, 2006, submitted. [Online]. Available: http://arxiv.org/math.OC/0601424

[10] A. E. Bryson and Y.-C. Ho, Applied Optimal Control. Hemisphere Publishing Corporation, 1975.

[11] C. T. Kelley, Iterative Methods for Linear and Nonlinear Equations. SIAM, 1995

[12] J. M. A. Danby, Fundamentals of Celestial Mechanics. Willmann Bell Inc., 1988.

[13] J. T. Betts, Practical Methods for Optimal Control Using Nonlinear Programming. SIAM, 2001. 